\title{
ON THE INITIAL DISTANCE OF THE MOON FORMING IN THE CIRCUMTERRESTRIAL SWARM
}

\author{
E. L. RUSKOL \\ O. Yu. Schmidt Institute of Physics of the Earth, Academy of Sciences, Moscow, U.S.S.R.
}

\begin{abstract}
According to the Radzievskij-Artemjev hypothesis of the 'locked' revolution of the circumplanetary swarms around the Sun, the initial Moon-to-Earth distance and the angular momentum acquired by the Earth through the accretion of the inner part of the swarm can be evaluated. Depending on the concentration of the density to the centre of the swarm we obtain the initial distance for a single protomoon in the range 15-26 Earth radii $R$ and for a system of 3-4 protomoons in the range 3-78 $R$, if the outer boundary of the swarm equals to the radius of the Hill's sphere ( $235 R)$. The total angular momentum acquired by the primitive Earth-Moon system through the accretion of the swarm particles is $\frac{1}{2}-\frac{2}{3}$ of its present value. The rest of it should be acquired from the direct accretion of interplanetary particles by the Earth. The contribution of satellite swarms into the rotation of other planets is relatively less.
\end{abstract}

The initial distance of the Moon forming in the circumterrestrial swarm of small Earth's satellites depends on the geocentric angular momentum of the swarm. The calculation of the value of that momentum was never done because of the great difficulty. The swarm may be imagined as a nearly spherical cluster of particles on initially chaotic orbits which gradually evolves into a regular disk-like system and then coagulates into one or several large satellites. It is of interest to evaluate the initial geocentric angular momentum of the Moon using the Radzievskij-Artemjev hypothesis of the 'locked' prograde revolution of the circumterrestrial swarm in respect to the Sun [1,2]. It is based on a supposition that the peculiar components of the remnant velocities of the particles captured into the swarm, are symmetric in the frame rotating with the Earth around the Sun. In this case the total geocentric angular momentum of the swarm equals $+I \omega_{c}$, where $I$ is the moment of inertia of the swarm relative to the axis going through the Earth and parallel to the axis of the ecliptics; $\boldsymbol{\omega}_{c}$ - the angular velocity of the Earth's motion around the Sun. (Let us remind for a comparison that every flat eddy in the circumsolar cloud of particles moving on keplerian circular orbits has the angular momentum $+\frac{1}{4} I \omega_{c}$ relative to the axis going through the geometric center of the eddy and parallel to the axis of rotation of the cloud.)

Suppose that all new particles captured into the swarm on the distance $l$ from the axis, in average have the specific angular momentum $l^{2} \omega_{c}$, corresponding to the 'locked' ('solid-body') rotation of the swarm on that distance. Inside the whole sphere of action of the Earth such a 'solid-body' momentum is much less than the geocentric angular momentum $\sqrt{ }(\mathrm{Gml})$ of a satellite on a direct circular orbit in the equatorial plane of the swarm. Therefore the conversion of the 'solid-body' swarm into a satellite or a satellite system must be accompanied by the contraction and by fall out of its inner part onto the Earth.

Let us denote the boundaries of the inner and outer parts of the swarm correspondingly by $\left(l_{1}, l_{2}\right),\left(l_{2}, l_{3}\right)$. Taking the dependence of the surface density of the swarm 
on the distance $l$ in the form $\sigma(l)=\sigma_{0}\left(l /\left(l_{1}+1\right)\right)^{-b}$, we can evaluate the ratio of the mass $M_{1}$ falling onto the Earth to the mass $\mu$ of the satellite:

$$
M_{1}=\frac{\int_{l_{1}}^{l_{2}} 2 \pi \sigma l \mathrm{~d} l}{l_{3} 2 \pi \sigma l \mathrm{~d} l}=\frac{l_{2}^{2-b}-l_{1}^{2-b}}{l_{3}^{2-b}-l_{2}^{2-b}}
$$

and the ratio of the corresponding angular momenta:

$$
\frac{K_{M_{1}}}{K_{\mu}}=\frac{\int_{l_{1}}^{l_{2}} \omega_{c} \cdot 2 \pi \sigma l^{3} \mathrm{~d} l}{\int_{l_{2}}^{l_{3}} \omega_{c} \cdot 2 \pi \sigma l^{3} \mathrm{~d} l}=\frac{l_{2}^{4-b}-l_{1}^{4-b}}{l_{3}^{4-b}-l_{2}^{4-b}} .
$$

The specific angular momentum of the satellite matter will be:

$$
k_{\mu}=\frac{K_{\mu}}{\mu}=\frac{\omega_{c}\left(l_{3}^{4-b}-l_{2}^{4-b}\right)(2-b)}{\left(l_{3}^{2-b}-l_{2}^{2-b}\right)(4-b)} .
$$

Taking $\mu=\mu_{\diamond}$, we can evaluate $K_{M_{1}}$, the angular momentum transferred to the Earth from the swarm.

The inner boundary of the swarm may be taken on the axis of rotation $\left(l_{1}=0\right)$, the outer boundary $l_{3}$ - the Hill's sphere radius $r_{1}=\mathfrak{R}\left(m / 3 M_{\odot}\right)^{1 / 3}$. For the present mass $M$ of the Earth it equals $235 R$, for $m=M / 2$ - nearly $190 R, R$ being the present Earth's radius. The boundary between the inner and the outer portions of the swarm, $l_{2}$, may be put $\approx 100 R$ from the condition that all circular orbits of prograde satellites should lie outside the Roche limit $\left(l^{\prime} \gtrsim 3 R\right)$.

The distribution of masses and angular momenta between the inner and outer parts of the swarm bounded by the Hill's sphere of radius $235 R$ is represented in the Table 1 for different values of $b$. The change of $b$ from 0 to 3 corresponds to a very significant change of concentration of the density to the center: from a uniform distribution of the surface density $\sigma$ over the swarm to the variation of $\sigma$ by 7 orders of magnitude within the swarm. This manifests in the great variation of the ratio $M_{1} / \mu$. Meanwhile the specific angular momentum of the satellite matter $k_{\mu}$ changes only from $2.6 \times 10^{15}$ to $1.9 \times 10^{15} \mathrm{cgs}$ which corresponds to the distance of a single protomoon in the interval 15-26 $R$. For a 3-4 protomoon system the distance interval is greater: from 3 to $78 R$. The total momentum transferred to the Earth-Moon system by the swarm, $K_{M_{1}}+K_{\mu}$, is always less than the present one $\left(3.45 \times 10^{41} \mathrm{cgs}\right)$; it reaches roughly $\frac{1}{2}-\frac{1}{3}$ of the present value. This result is in agreement with the R.T. Giuli's conclusion that a considerable part of the momentum of the Earth-Moon system (up to $\frac{1}{2}$ ) is brought to the Earth by particles from heliocentric orbits [3]. The initial distance of the Moon 


\section{TABLE I}

The distribution of the mass and the angular momentum between the inner and the outer parts of the swarm inside the Hill's sphere $(235 R)$

\begin{tabular}{llllll}
\hline$b$ & 0 & 1 & 2 & 2.5 & 3 \\
\hline$M_{1} / \mu$ & 0.22 & 0.73 & 5.4 & 25.8 & 172 \\
$\mathrm{~K}_{M_{1}} / K_{\mu}$ & 0.033 & 0.083 & 0.22 & 0.38 & 0.73 \\
$k_{M_{1}}$ cgs & $4 \times 10^{14}$ & $2.7 \times 10^{14}$ & $8.7 \times 10^{13}$ & $3 \times 10^{13}$ & $8 \times 10^{12}$ \\
$k_{\mu}$, cgs & $2.6 \times 10^{15}$ & $2.4 \times 10^{15}$ & $2.1 \times 10^{15}$ & $2 \times 10^{15}$ & $1.9 \times 10^{15}$ \\
$K_{M_{1}}$ at $\mu=\mu_{\varangle}$ & $6.5 \times 10^{39}$ & $1.4 \times 10^{40}$ & $3.4 \times 10^{40}$ & $5.6 \times 10^{40}$ & $1 \times 10^{41}$ \\
$K_{\mu}$ at $\mu=\mu_{\varangle}$ & $1.9 \times 10^{41}$ & $1.75 \times 10^{41}$ & $1.55 \times 10^{41}$ & $1.46 \times 10^{41}$ & $1.4 \times 10^{41}$ \\
$K_{M_{1}}+K_{\mu}$ & $2 \times 10^{41}$ & $1.9 \times 10^{41}$ & $1.9 \times 10^{41}$ & $2.1 \times 10^{41}$ & $2.4 \times 10^{41}$ \\
\hline
\end{tabular}

The angular momentum of the Earth acquired by the direct accretion after Giuli [3] $K_{M_{0}}=1.7-1.8 \times 10^{41}$. Theoretical sum $K_{M_{0}}+K_{M_{1}}+K_{\mu}=3.7 \times 10^{41}$.

The present value $K_{M+\mu}=3.45 \times 10^{41} \mathrm{cgs}$.

depends considerably upon the choice of the outer boundary of the swarm. For the outer boundary $190 R$, for example, all satellite orbits occur inside the distance $30 R$, and the orbit of a single protomoon - at 10-15 $R$. These results do not contradict the conclusion by $\mathrm{P}$. Goldreich that the most plausible zone of accumulation of the Moon is the interval 10-30 [4].

The plausible initial distances of the Moon may be considered as an argument in favor of the 'solid-body' swarm filling the Hill's sphere around the growing Earth.

\section{References}

[1] Artemjev, A. V. and Radzievskij, V. V.: 1965, Astron. Zh. 42, 124.

[2] Artemjev, A. V.: 1963, Ann. Faroslavl Pedagogical Inst., ser. Astronomia, issue 56.

[3] Giuli, R. T.: 1968, Icarus 8, 301.

[4] Goldreich, P.: 1966, Rev. Geophys. 4, 411. 